\section{The Effects of Signal Contamination on Two Adaptive Detectors}

KARL GERLACH, Member, IEEE

Naval Research Laboratory

Two schemes for adaptive detection, Kelly's generalized likelihood ratio test (GLRT) and the mean level adaptive detector (MLAD), are analyzed with respect to the deleterious effect of desired-signal contamination of the data used to compute the sampled covariance matrix for the two detectors. The detection probability $P_{D}$ and false alarm performance (ghosting probability $P_{G}$ ) are predicted for the two schemes under the assumptions that the input noises are Gaussian random variables that are temporally independent but spatially correlated; and the amplitude of the desired signal is Rayleigh distributed. $P_{D}$ and $P_{G}$ are computed as a function of the false alarm probability $P_{F}$ with no contamination, the number of input channels, the number of independent samples-per-channel, the matched filtered output signal-to-noise $(S / N)$ power ratio, and the $S / N$ power ratio of the contaminating desired signal. It is shown that both $P_{D}$ and $P_{G}$ decrease with increasing levels of contamination. The $P_{G}$ performance is almost identical for the GLRT and MLAD. The $P_{D}$ performance shows similar relative performance trends. Significantly, it is shown that the ghosting probability does not exceed $P_{F}$ in the presence of contamination.

Manuscript received March 2, 1993; revised September 7 and October 26, 1993.

IEEE Log No. T-AES/31/1/08010.

Author's address: Naval Research Laboratory, Radar Division, Code 5341, Washington, DC 20375-5000.

U.S. Government work. Not protected by U.S. copyright.

$0018-9251 / 95 / \$ 4.00$

\section{INTRODUCTION}

In [1], two schemes for adaptive detection were compared: Kelly's generalized likelihood ratio test (GLRT) [2] and the mean level adaptive detector (MLAD). Detection performance $P_{D}$ was predicted for the two schemes under the assumptions that the input noises are zero-mean Gaussian random variables (RVs) that are temporally independent but spatially correlated, and that the amplitude of the desired signal is Rayleigh distributed. $P_{D}$ was computed as a function of the false alarm probability, the number of input channels, the number of independent samples-per-channel, and the matched filtered output signal-to-noise $(S / N)$ power ratio. The GLRT was shown to have better detection performance than the MLAD. The difference in detection performance increased as one used fewer input samples; however, the required number of samples necessary to have only a $3 \mathrm{~dB}$ detection loss for both detection schemes is approximately the same. This is significant since, for the present, the MLAD is considerably less complex to implement than the GLRT.

The general problem of signal detection in a background of Gaussian noise for an adaptive array was first addressed by Kelly [2] by using the techniques of statistical hypothesis testing. In [2], the problem is formulated as a binary hypothesis test where one hypothesis is noise only and the other is signal-plus-noise. A given input data vector (called the primary data vector) is tested for signal presence. Another set of signal-free data vectors (called the secondary data vectors) is available that shares the unknown covariance matrix $\mathbf{M}$ of the noise in the primary data vector. A likelihood ratio decision rule was derived, and its performance was evaluated for the two hypothesis.

Kelly's detector uses the maximum likelihood (ML) estimates for the unknown parameters of the likelihood ratio test (LRT). The unknown parameters are the spatial covariance matrix and the complex amplitude of the unknown signal (assumed in Kelly's analysis to be a nonrandom constant). This detection scheme is commonly referred to as the GLRT.

A less complicated adaptive detection scheme is found by implementing the MLAD. The MLAD is essentially an adaptive matched filter (AMF) followed by a mean level detector (MLD) [3, 4]. Input samples used in determining the MLD threshold are derived from a block of data passing through the AMF. This same block of data is used to calculate the AMF weights. The squared magnitude of each of these same samples as processed through the AMF is used as a test statistic and compared against an MLD threshold (an average of the instantaneous powers) that does not contain the given test statistic sample. We further clarify the implementation terminology by calling this an MLAD with concurrent data samples. 
In [5], an analysis was performed for an MLAD with nonconcurrent data, i.e., the block of data that passes through the AMF that is used to determine the MLD threshold is statistically independent of the block of data used to calculate the AMF weights. Other work pertinent to this paper is Wang and Cai [6] who developed the theory of using a multiple adaptive matched filter bank detector (embodied by the modified sample matrix inversion algorithm (MSMIA)) and multiband GRLT test. These tests have application in detecting a target of unknown velocity in clutter using a matched Doppler filter bank/detector configuration.

We note that under the above stated assumptions for both Kelly's GLRT and the MLAD, the $P_{F}$ does not depend on $\mathbf{M}$ (a second-order characterization of the external noise environment). Hence these detectors exhibit the desirable constant false alarm rate (CFAR) property of having the $P_{F}$ be independent of the covariance matrix.

Here we extend the results of [1] to include the effects of what we term "desired-signal contamination." By this, we mean that a significant level of a desired signal is present in the secondary data vectors, which, for both Kelly's GLRT and the MLAD, are used to estimate the unknown covariance matrix $\mathbf{M}$. We assume that the contaminating desired signal is statistically independent of the desired signal in the primary data vector. In practice, contamination can be caused by a variety of mechanisms, such as for the radar problem, multiple target returns being present at different ranges of the pulse compressor output. In this case, a number of the multiple target returns at distinct times that have the waveform of the desired signal may be in the secondary data.

For our analysis, we simplify the contamination model by only considering a single source of contamination. In addition, this single source contaminates only one sample vector of the secondary data vectors. Two performance measures are affected: $P_{D}$ and $P_{F}$. Because $P_{F}$ is normally chosen under the condition of no contamination, we redesignate the probability of false alarm in the presence of contamination as the ghosting probability $P_{G}$. We do this since ghosts (in the radar sense) are desired signal induced false detections.

Here we present results on the detection and ghosting performance of GLRT and MLAD in the presence of contamination. As in [1], we assume that the desired signal is a complex zero-mean Gaussian RV of unknown variance with independent and identically distributed (IID) real and imaginary parts (the magnitude of this amplitude is Rayleigh distributed). Under the GLRT, we would have to reformulate Kelly's detector with the variance of the unknown signal amplitude as an unknown parameter, and find the ML estimate of this quantity. This proved to be mathematically tedious. In lieu of implementing this new GLRT, we choose to evaluate Kelly's GLRT, as it defined in his paper. As noted by Kelly, no optimality properties are claimed for this test. The form of the test is, however, reasonable.

\section{GENERALIZED LIKELIHOOD RATIO TEST}

\section{A. Detector Form}

A mathematical formulation of the adaptive detection problem that leads to the GLRT is given by Kelly [2]. We now summarize that formulation. Two sets of input data are used, called the primary and secondary inputs. The secondary inputs are assumed not to contain the desired signal. Set

$X=N \times K$ complex matrix of secondary input data. The $n$th row represents the $K$ samples of data on the $n$th channel, where $n=1,2, \ldots, N$. The samples in the $k$ th column are assumed time-coincident.

$\mathbf{x}=$ primary data vector (complex) of length $N$. $\mathbf{s}=$ desired steering vector (complex) of length $N$.

Consider the two hypothesis:

$$
\begin{aligned}
& H_{0}: \mathbf{x}=\mathbf{n} \\
& H_{1}: \mathbf{x}=\mathbf{n}+a \mathbf{s}
\end{aligned}
$$

where $H_{0}$ is the noise only hypothesis, $\mathbf{n}$ is a noise vector of length $N, H_{1}$ is the signal-plus-noise hypothesis, and $a$ is the unknown complex signal amplitude. We make the following assumptions.

A1) Input noises are complex zero-mean stationary Gaussian RVs. The real and imaginary parts of a given input noise sample are IID with respect to each other (an RV with these characteristics is called a circular Gaussian process).

A2) Input noise samples are temporally statistically independent.

A3) The secondary data is statistically independent of the primary data.

A4) $K \geq N$.

The GLRT is formulated as follows. The joint probability density function (pdf) under each hypothesis over all measured data is found. For this problem, this is straightforward, since the sample vectors are assumed independent and each vector has an associated $N$-dimensional Gaussian pdf. If there are any unknown parameters, the pdf of the inputs is maximized over all unknown parameters separately for each of the two hypotheses. The maximizing parameter values are by definition the ML estimators of the parameters. Hence the maximized pdfs are obtained by replacing the unknown parameters by their ML estimates. The ratio of the resultant maximum of pdfs is found (the ratio of the pdf under $H_{1}$ to the pdf 
under $H_{0}$ ). This ratio is checked to see if it exceeds a preassigned threshold $t$.

Kelly shows that the GLRT for the adaptive detection problem is given by

$$
\frac{\left|\mathbf{s}^{\mathrm{H}} \hat{R}_{x}^{-1} \mathbf{x}\right|^{2}}{\left(\mathbf{s}^{\mathrm{H}} \hat{R}_{x}^{-1} \mathbf{s}\right)\left[1+\mathbf{x}^{\mathrm{H}} \hat{R}_{x}^{-1} \mathbf{x}\right]} \stackrel{H_{1}}{\gtrless} t
$$

where

$$
\hat{R}_{x}=X X^{\mathrm{H}}
$$

and $\mathrm{H}$ denotes the conjugate transpose matrix operation. We note that $\hat{R}_{x}$ is proportional to the ML estimate of the input covariance matrix. We note also that the unknown complex amplitude $a$ of the desired signal has been estimated and is accounted for in (3). The elements of $\hat{R}_{x}$ are RVs that are functions of the input samples (the elements of $X$ ). It is straightforward to show that the probabilistic measure of the set of $X$, for which $\hat{R}_{x}$ is singular, is zero. Hence, when assessing detection performance, we can always assume that $\hat{R}_{x}$ (or any other matrix that has the form given by (4)) is invertible.

For the signal contamination model, we make the following additional assumption.

A5) A statistically independent desired signal is always present in the secondary data under $H_{0}$ or $H_{1}$. It is only present on the $K$ th sample vector (the $K$ th column of $X$ ).

For A5, the choice of which secondary sample vector in time is contaminated is arbitrary and does not affect the analysis. In practice, this contamination is quite common when more than one target return is contained within the processing window for adaptation. One target return is in the candidate detection cell and the other (from a different target in a different range cell) is in the secondary data.

\section{B. Statistically Equivalent GLRT}

In [1], a statistically equivalent GLRT was derived that was used to formulate in simple fashion the $P_{D}$ and $P_{F}$ probabilities of the adaptive detector with no signal contamination. With one modification of the development in [1], we can derive a statistically equivalent GLRT that allows us to formulate the $P_{D}$ and $P_{G}$ for the adaptive detector with signal contamination (which is modeled as given by A5). We briefly outline the methodology used to obtain the statistically equivalent GLRT.

As in Kelly's (and Reed, Mallet, and Brennan [7]) development, we can matrix transform the input vectors by an $N \times N$ matrix $A$, which has the properties that the input noise vectors are spatially whitened, each input element has noise power normalized to one, and

$$
A \mathbf{s}=\left(0,0, \ldots 0,\left(\mathbf{s}^{\mathrm{H}} M^{-1} \mathbf{s}\right)^{1 / 2}\right) \equiv \mathbf{s}_{0}
$$

where all of the desired signal has been placed into $N$ th channel (note that in Kelly's paper, the signal was placed into the first channel; for our analysis, we place the signal into the $N$ th channel).

In addition, set

$$
\begin{aligned}
& \mathbf{z}=A \mathbf{x} \\
& Z=A X .
\end{aligned}
$$

The element of vector $\mathbf{z}$ (under $H_{0}$ ) and the elements of the vectors representing the columns of $Z$ (each column represents the transformed secondary data across the array at a given instant of time) are now spatially independent with each element having power equal to 1. As shown by Kelly, the transformed GLRT is given by

$$
\frac{\left|\mathbf{s}_{0}^{\mathrm{H}} \hat{R}_{z}^{-1} \mathbf{z}\right|^{2}}{\left(\mathbf{s}_{0}^{\mathrm{H}} \hat{R}_{z}^{-1} \mathbf{s}_{0}\right)\left(1+\mathbf{z}^{\mathrm{H}} \hat{R}_{z}^{-1} \mathbf{z}\right)} \stackrel{H_{1}}{\gtrless} t
$$

where

$$
\hat{R}_{z}=Z Z^{\mathrm{H}} .
$$

We note that the desired-signal contamination of the secondary data is completely contained in the $N, K$ element of $Z$.

In [1], we show that by using a series of unitary matrix transformations $(K \times K$ transforms on $Z$ and an $N \times N$ transform on $\mathbf{z}$ ), the following statistically equivalent GLRT results:

$$
\left|u_{11} \nu_{2}-\nu_{1} u_{21}\right|^{2} \underset{H_{0}}{\stackrel{H_{1}}{\gtrless}}\left(u_{11}^{2}+\nu_{1}^{2}\right) u_{22}^{2} T
$$

where

$$
T=\frac{t}{1-t}
$$

and $u_{11}, u_{21}, u_{22}, \nu_{1}$, and $\nu_{2}$ are independent RVs. Furthermore, $u_{11}$ has the $\chi$ pdf of order $2(K-N)$ with $\sigma^{2}=0.5, \nu_{1}$ has the $\chi$ pdf of order $2(N-1)$ with $\sigma^{2}=0.5$ under $H_{1}, \nu_{2}$ is the sum of the desired signal in the primary data plus a circular Gaussian RV with power equal 1 , and $u_{21}$ is a circular Gaussian RV with power equal 1. In addition, if we adopt the notation of [1], then

$$
u_{22}^{2}=\sum_{k=N}^{K}\left|z_{N k}^{(N-1)}\right|^{2}
$$

where $z_{N k}^{(N-1)}, k=N, N+1, \ldots, K-1$ are IID circular Gaussian RV with power equal 1 and $z_{N K}^{(N-1)}$ is the sum of the contaminating desired signal plus a circular Gaussian RV with power equal 1. Hence, the desired signal contamination which was originally contained in the last column of the secondary data matrix $X$, through a series of orthogonal transformations is completely contained in the $N, K$ element of $Z$ and 
enters the statistical analysis via the last term of the summation given by (11).

We use the statistically equivalent GLRT given by (9) and the aforementioned probabilistic characterizations of $u_{11}, u_{21}, u_{22}, \nu_{1}$, and $\nu_{2}$ as the starting point for our analysis.

\section{Probability of Detection}

Under the $H_{1}$ hypothesis, we assume that the desired signal amplitude of the primary vector is Rayleigh distributed and the phase of the signal is uniformly distributed between $(0,2 \pi)$. This implies that the desired signal itself is a complex circular Gaussian RV. Let the desired signal's input power-per-channel before any matrix transformation be equal to $\tilde{\sigma}_{s}^{2}$. After the $A$ matrix transformation (whitening, normalizing, and placing the signal into the $N$ th channel), the signal power in the $N$ th channel is $\sigma_{s}^{2}=\tilde{\sigma}_{s}^{2} \mathbf{s}^{\mathrm{H}} \mathbf{M}^{-1} \mathbf{s}$. Thus under $H_{1}, \nu_{2}$ is a complex circular Gaussian RV with power equal to $\sigma_{s}^{2}+1$, where the 1 represents the noise power-level-per-channel after the $A$ matrix transformation. In similar fashion, let the contaminating desired signal's input power-per-channel before any matrix transformation be equal to $\tilde{\sigma}_{c}^{2}$. Again we assume that the amplitude of the contaminating desired signal is Rayleigh distributed with phase uniformly distributed between $(0,2 \pi)$. After the $A$ transformation, $\sigma_{c}^{2}=\tilde{\sigma}_{c}^{2} \mathbf{s}^{H} \mathbf{M}^{-1} \mathbf{s}$. Thus under $H_{0}$ or $H_{1}, z_{N K}^{(N-1)}$ is a complex circular Gaussian RV with power equal to $\sigma_{c}^{2}+1$, where again the 1 represents the noise power-level-per-channel after the $A$ matrix transformation.

Recall that $u_{21}$ is a complex circular Gaussian RV with power equal to 1 . We can rewrite (9) as

$$
|\alpha|^{2} \underset{H_{0}}{\stackrel{H_{1}}{\gtrless}} T^{\prime}
$$

where

$$
\begin{aligned}
\alpha & =\frac{u_{11} \nu_{2}-\nu_{1} u_{21}}{\left(u_{11}^{2}\left(\sigma_{s}^{2}+1\right)+\nu_{1}^{2}\right)^{1 / 2}} \\
T^{\prime} & =\frac{u_{11}^{2}+\nu_{1}^{2}}{u_{11}^{2}\left(\sigma_{s}^{2}+1\right)+\nu_{1}^{2}} u_{22}^{2} T .
\end{aligned}
$$

It is straightforward to show that $\alpha$, when conditioned on $u_{11}$ and $\nu_{1}$, is a complex circular Gaussian RV with power equal to 1 . It is well known [8] that the conditional probability of detection is given by

$$
P_{D}\left(u_{11}, u_{22}, \nu_{1}\right)=e^{-T^{\prime}}=\exp \left\{-\frac{u_{11}^{2}+\nu_{1}^{2}}{u_{11}^{2}\left(\sigma_{s}^{2}+1\right)+\nu_{1}^{2}} u_{22}^{2} T\right\}
$$

where $P_{D}(\cdot)$ denotes the probability of detection conditioned on the arguments $\cdot$.
We set

$$
\begin{aligned}
& \eta=u_{22}^{2}, \\
& \mu=u_{11}^{2}, \\
& \nu=\nu_{1}^{2}, \\
& r=\frac{\nu}{\mu} .
\end{aligned}
$$

Then (15) becomes

$$
P_{D}(r, \eta)=\exp \left\{-\frac{1+r}{\sigma_{s}^{2}+1+r} \eta T\right\} .
$$

The pdfs of $\mu$ and $\nu$ are $\chi^{2}$ of order $2(K-N+2)$ and $2(N-1)$, respectively, with $\sigma^{2}=0.5$ and are given by

$$
\begin{aligned}
& p_{\mu}(\mu)=\frac{1}{(K-N+1) !} \mu^{K-N+1} e^{-\mu}, \quad \mu \geq 0 \\
& p_{\nu}(\nu)=\frac{1}{(N-2) !} \nu^{N-2} e^{-\nu}, \quad \nu \geq 0 .
\end{aligned}
$$

The pdf of $\eta$ is derived in the Appendix and is given by

$$
\begin{array}{r}
P_{\eta}(\eta)=\frac{1}{\left(\sigma_{c}^{2}+1\right)(K-N-1) !} \eta^{K-N} e^{-\eta /\left(1+\sigma_{c}^{2}\right)} \\
\times \int_{0}^{1} \lambda^{K-N-1} \exp \left\{-\lambda \eta \frac{\sigma_{c}^{2}}{\sigma_{c}^{2}+1}\right\} d \lambda, \\
\eta \geq 0 .
\end{array}
$$

By using elementary probability theory, it is straightforward to show that

$$
p_{r}(r)=\int_{0}^{\infty} \frac{\beta}{r^{2}} p_{\nu}(\beta) p_{\mu}\left(\frac{\beta}{r}\right) d \beta .
$$

By inserting expressions for $p_{\mu}$ and $p_{\nu}$ as given by (21) and (22), respectively, and simplifying results in the following expression for $p_{r}$ :

$$
\begin{array}{r}
p_{r}(r)=\frac{K !}{(N-2) !(K-N+1) !} \frac{r^{N-2}}{(1+r)^{K+1}}, \\
r \geq 0 .
\end{array}
$$

If we set $q=1 /(1+r)$, it is straightforward to show that

$$
\begin{gathered}
p_{q}(q)=\frac{K !}{(N-2) !(K-N+1) !}(1-q)^{N-2} q^{K-N+1}, \\
0 \leq q \geq 1
\end{gathered}
$$

which is the Beta distribution derived by Reed, et al. [7] for the normalized instantaneous $S / N$ power 
ratio and which results if the sampled matrix inversion (SMI) algorithm is used. By substituting $q$ for $r$ in (20),

$$
P_{D}(q, \eta)=\exp -\left[\frac{1}{q \sigma_{s}^{2}+1}\right] \eta T .
$$

If we multiply (27) by the pdf of $\eta$ as given by (23) and integrate over the support of $\eta$, it is straightforward to show

$$
P_{D}(q)=\frac{K-N}{\sigma_{c}^{2}+1} \int_{0}^{1} \frac{\lambda^{K-N-1} d \lambda}{\left[\frac{T}{q \sigma_{s}^{2}+1}+\frac{1+\lambda \sigma_{c}^{2}}{\sigma_{c}^{2}+1}\right]^{K-N+1} .}
$$

Thus

$$
P_{D}=\int_{0}^{1} P_{D}(q) p_{q}(q) d q
$$

where $p_{q}(q)$ is given by (26). We set $(S / N)_{\mathrm{opt}}=\sigma_{s}^{2}$, where $(S / N)_{\text {opt }}$ is the optimal $S / N$ output power ratio of the matched filter $(K=\infty)$. We can write this in this way because the output noise power of the $N$ th channel has been normalized to 1 and the output of the $N$ th channel is the optimal matched filter output. We also set $(S / N)_{\text {con }}=\sigma_{c}^{2}$ where $(S / N)_{\text {con }}$ is the contaminated $S / N$ output power ratio of the matched filter $(K=\infty)$.

\section{Probability of Ghosting}

The probability of a ghost is easily derived from (28) and (29) by setting $\sigma_{s}^{2}=0$. It is found that

$$
P_{G}=\frac{K-N}{\sigma_{c}^{2}+1} \int_{0}^{1} \frac{\lambda^{K-N-1} d \lambda}{\left[T+\frac{1+\lambda \sigma_{c}^{2}}{1+\sigma_{c}^{2}}\right]^{K-N+1}} .
$$

If we set $\sigma_{c}^{2}=0$, then

$$
P_{G}=P_{F}=\frac{1}{(T+1)^{K-N+1}}
$$

which is the probability of false alarm for the GLRT derived in [1] under the condition that there is no contamination.

\section{MEAN LEVEL ADAPTIVE DETECTOR}

\section{A. Detector Form and Statistically Equivalent MLAD}

The hypothesis setup given by (1) and (2) applies for the forthcoming development. In [1], an MLAD was formulated for the adaptive detection problem. In this subsection, we briefly review this formulation for the MLAD and give a statistically equivalent form that is used to obtain results for $P_{D}$ and $P_{G}$.

An intuitive form of adaptive detection is found by implementing the MLAD. The MLAD is essentially an AMF followed by an MLAD, as illustrated in
Fig. 1. The MLAD is designed to perform detections over a block of data by using just this block of data in determining the AMF weights and the MLD threshold. The MLAD works as follows. Let there be $N$ channels and $K+1$ samples per channel. Define

$$
\begin{aligned}
& \mathbf{x}=\text { primary } N \text {-length data vector (complex). } \\
& \mathbf{x}_{k}=\text { secondary } N \text {-length data vector (complex), } \\
& k=1,2, \ldots, K \\
& \quad X_{\text {aug }}=(\mathbf{x} \mid X)=\text { augmented } N \times(K+1) \text { complex } \\
& \text { matrix of input data. } \\
& \hat{R}_{0}=X_{\text {aug }} X_{\text {aug }}^{\mathrm{H}} .
\end{aligned}
$$

The $N$-length weighting vector $\hat{\mathbf{w}}$ for the AMF is found by using the SMI algorithm and is given by

$$
\hat{\mathbf{w}}=\hat{R}_{0}^{-1} \mathbf{s}
$$

This weight is used in the detection rule given by

$$
\left|\hat{\mathbf{w}}^{\mathrm{H}} \mathbf{x}\right|^{2} \underset{H_{0}}{\stackrel{H_{1}}{\gtrless}} T_{0} \sum_{k=1}^{K}\left|\hat{\mathbf{w}}^{\mathrm{H}} \mathbf{x}_{k}\right|^{2}
$$

where $T_{0}$ is chosen to control the false alarm probability. We see that (32) is the algorithmic representation of the AMF and (33), the MLD.

Note that we have included the primary data vector in the $\hat{R}_{0}$ estimate and, hence, in the $\hat{\mathbf{w}}$ estimate. In a practical situation, this might be done, since it is more numerically efficient to compute one weighting vector over the entire data block than it is to compute a distinct weighting vector for each point in the block. However, the presence of the desired signal (under $H_{1}$ ) will affect detection. In (33), the primary data vector is varied across the $K+1$ data snapshots, where the $\mathbf{x}_{k}$ used on the right side of (33) does not include a selected primary data vector.

It was shown in [1] that a statistically equivalent MLAD is given by

$$
\begin{gathered}
\left|u_{11} \nu_{2}-\nu_{1} u_{21}\right|^{2}\left[1-T_{1}\left(1+\frac{\nu_{1}^{2}}{u_{11}^{2}}\right)\right] \\
\stackrel{H_{1}}{\gtrless}\left(1+\frac{\nu_{1}^{2}}{u_{11}^{2}}\right)^{2} u_{11}^{2} u_{22}^{2} T_{1}
\end{gathered}
$$

where $u_{11}, u_{21}, u_{22}, \nu_{1}$, and $\nu_{2}$ are as probabilistically characterized in Section IIB and $T_{1}=T_{0} /\left(T_{0}+1\right)$.

We note for $T_{1}>0$ that if

$$
1-T_{1}\left(1+\frac{\nu_{1}^{2}}{u_{11}^{2}}\right) \leq 0
$$

then $H_{0}$ is declared.

\section{B. Probability of Detection}

Again under the $H_{1}$ hypothesis, we assume the desired signal of the primary vector and the contaminating desired signal are complex circular Gaussian RV (the amplitude is Rayleigh distributed). 
SENSORS

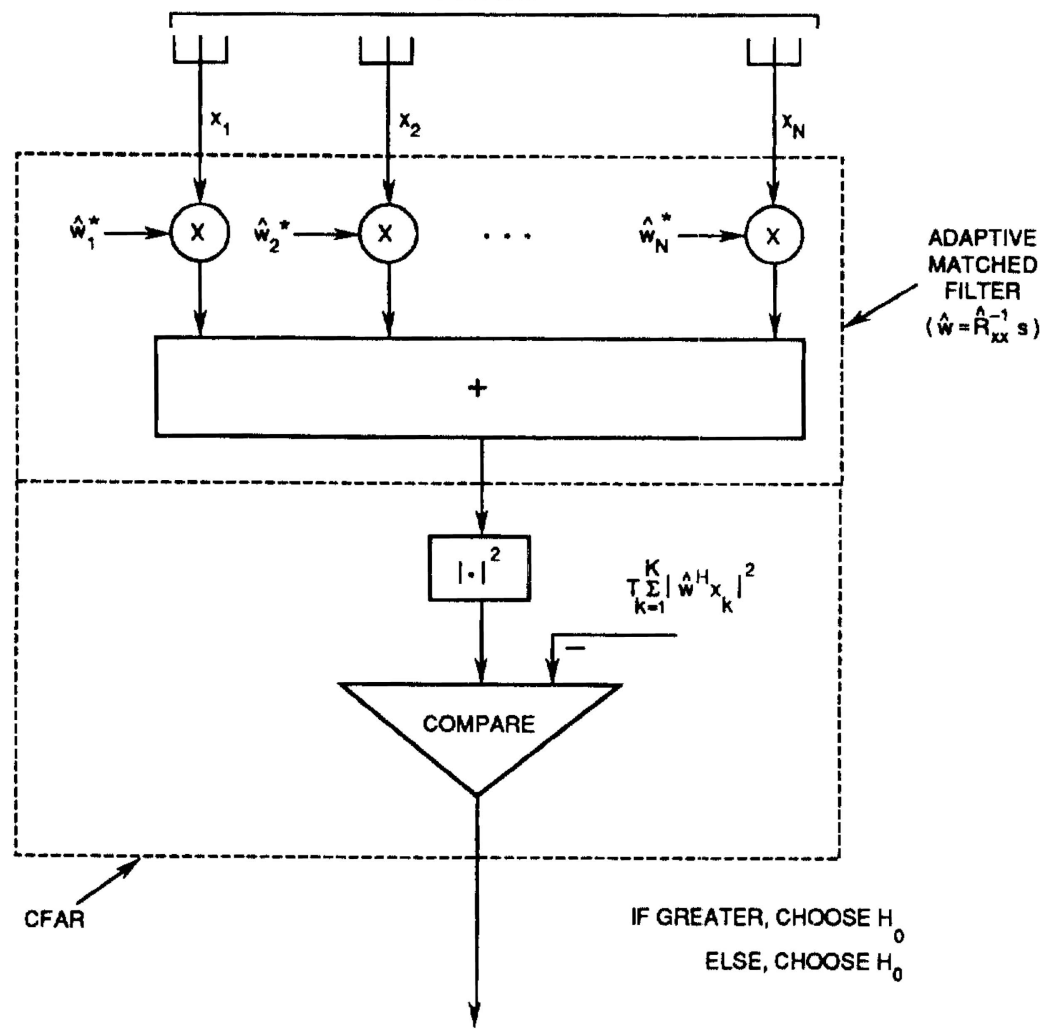

Fig. 1. MLAD.

As in our analysis of the GLRT, $\sigma_{s}^{2}=\tilde{\sigma}_{s}^{2} \mathbf{s}^{\mathrm{H}} \mathbf{M}^{-1} \mathbf{s}$ and $\sigma_{c}^{2}=\tilde{\sigma}_{c}^{2} \mathbf{s}^{\mathrm{H}} \mathbf{M}^{-1} \mathbf{s}$. Assume (35) is not true. We can write the decision rule given by (34) as

$$
|\alpha|^{2} \underset{H_{0}}{\stackrel{H_{1}}{\gtrless}} T_{1}^{\prime}
$$

where

$$
\begin{aligned}
\alpha & =\frac{u_{11} \nu_{2}-\nu_{1} u_{21}}{\left(u_{11}^{2}\left(\sigma_{s}^{2}+1\right)+\nu_{1}^{2}\right)^{1 / 2}} \\
T_{1}^{\prime} & =\frac{1}{\left(q-T_{1}\right)\left(q \sigma_{s}^{2}+1\right)} \eta T_{1}
\end{aligned}
$$

with $\eta$ defined by (16), and $q=(1+r)^{-1}$. As before, under $H_{1}, \nu_{1}$ is a complex circular Gaussian RV with power equal to $\sigma_{s}^{2}+1$, and $u_{21}$ is the same with power equal to 1 . Furthermore, $\alpha$ is the same with power equal to 1 . Thus

$$
P_{D}(q, \eta)= \begin{cases}0, & \text { if } q \leq T_{1} \\ e^{-T^{\prime}}, & \text { otherwise. }\end{cases}
$$

The PDFs of $\eta$ and $q$ are given by (23) and (26), respectively. If we multiply $P_{D}(q, \eta)$ by $p_{\eta}(\eta)$ and integrate over the support of $\eta$, after some simplification, it is found that for $q>T_{1}$,

$$
P_{D}(q)=\frac{K-N}{\sigma_{c}^{2}+1} \int_{0}^{1} \frac{\lambda^{K-N-1} d \lambda}{\left[\frac{T_{1}}{\left(q-T_{1}\right)\left(q \sigma_{s}^{2}+1\right)}+\frac{1+\lambda \sigma_{c}^{2}}{1+\sigma_{c}^{2}}\right]^{K-N+1} .}
$$

Because $P_{D}(q)=0$ for $q \leq T_{1}$, it follows that

$$
P_{D}=\int_{T_{1}}^{1} P_{D}(q) p_{q}(q) d q,
$$

where $p_{q}(q)$ is given by (26). Again we set $(S / N)_{\mathrm{opt}}=$ $\sigma_{s}^{2}$ and $(S / N)_{\text {con }}=\sigma_{c}^{2}$.

\section{Probability of Ghosting}

The $P_{F}$ is found by setting $(S / N)_{\mathrm{opt}}=0$ in (40). The following equations result:

$$
P_{G}(q)= \begin{cases}\frac{K-N}{\sigma_{c}^{2}+1} \int_{0}^{1} \frac{\lambda^{K-N-1} d \lambda}{\left[\frac{T_{1}}{q-T_{1}}+\frac{1+\lambda \sigma_{c}^{2}}{1+\sigma_{c}^{2}}\right]^{K-N+1},} & q>T_{1} \\ 0, & q \leq T_{1}\end{cases}
$$

and

$$
P_{G}=\int_{T_{1}}^{1} P_{G}(q) p_{q}(q) d q .
$$

If we set $\sigma_{c}^{2}=0$, it is straightforward to show that

$$
P_{G}=P_{F}=\left(1-T_{1}\right)^{K}
$$

which is the false alarm probability of the MLAD derived in [1] under the condition that there is no contamination. 


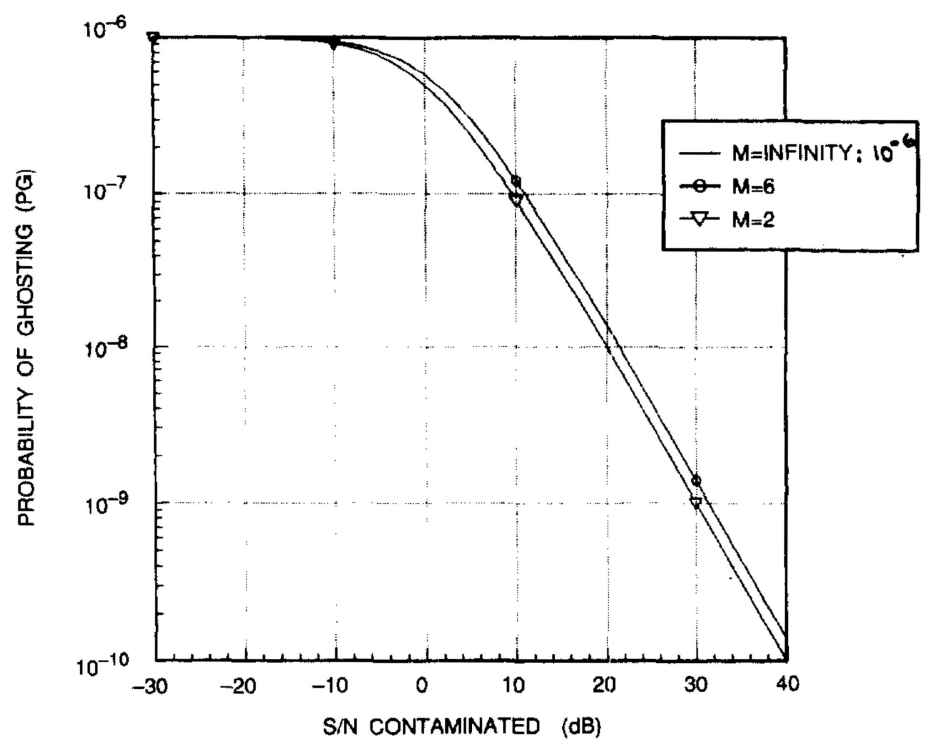

Fig. 2. Kelly detector: $P G$ for contaminated signal $N=2, P F=1$.D-6.

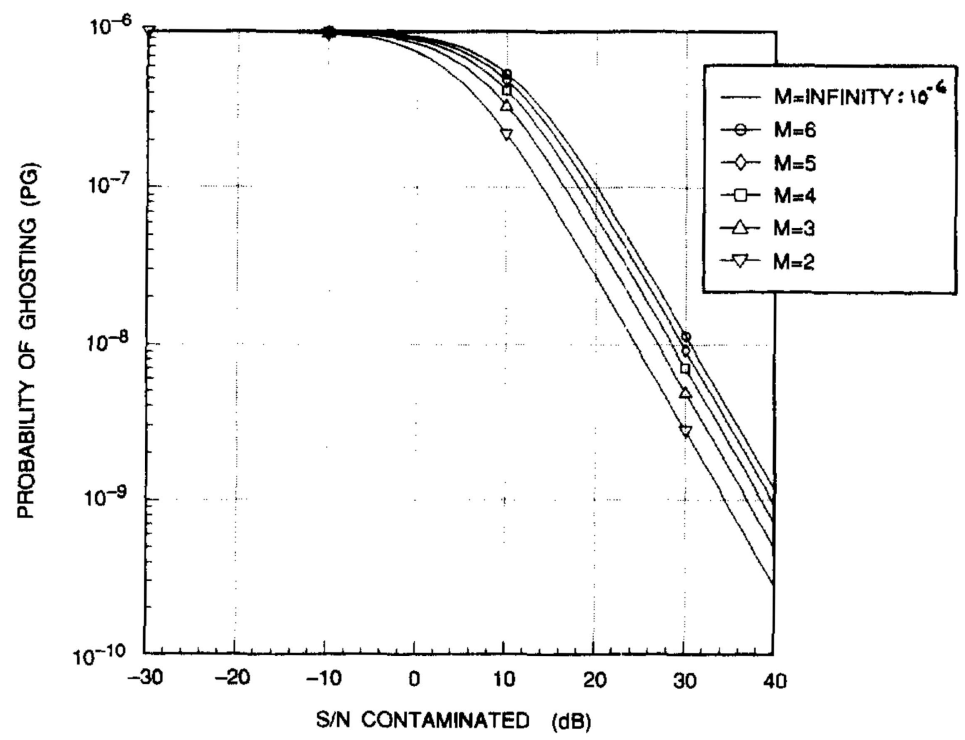

Fig. 3. Kelly detector: $P G$ for contaminated signal $N=30, P F=1$.D-6.

\section{RESULTS}

Here we present results for the detection probability $P_{D}$ in contamination and ghosting probability $P_{G}$ for the GLRT and MLAD versus the independent parameters: the quiescent probability of false alarm (when there is no contamination) $P_{F}$; the steady state $(K=\infty) S / N$ output power ratio of the matched filter $(S / N)_{\text {opt }}$; the contaminated $S / N$ power ratio $(S / N)_{\text {con }}$; the number of independent samples per channel $K$ of secondary data; and the number of input channels $N$. We set $K=M N$, where $M$ is a positive integer $\geq 2$, and use $M$ instead of $K$ as an independent parameter called the degrees-of-freedom factor.

Because there are many independent parameters, we present results for a representative set as shown in Figs. 2-15. Here we plot $P_{G}$ versus $(S / N)_{\text {con }}$ for $N=2,30 ; P_{F}=10^{-6}, 10^{-10}$, and $M=2,3, \ldots, 6$. For plotting $P_{D}$, we choose $(S / N)_{\text {opt }}$ to equal either $20 \mathrm{~dB}$ or $30 \mathrm{~dB}$, where a given $(S / N)_{\text {opt }}$ will yield a steady state $(K=\infty) P_{D}$, which is indicated by the horizontal line in Figs. 6-15. For plotting $P_{G}$ in the steady state, $P_{G}=P_{F}$, which is indicated by the horizontal line in Figs. 2-5.

We found that the $P_{G}$ performance results were identical for both the GLRT and MLAD and a given set of input parameters. Thus, we give only the $P_{G}$ performance results for the GLRT. Some pertinent observations to be made from Figs. 2-15 follow.

1) Both $P_{D}$ and $P_{G}$ degrade monotonically with increasing contamination $(S / N)_{\text {con }}$. 


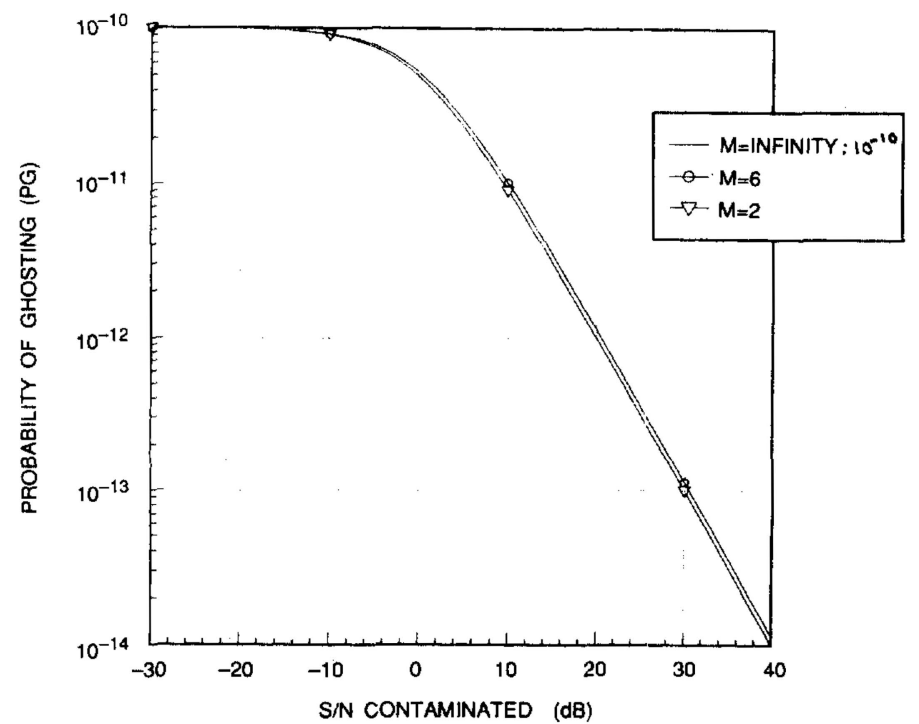

Fig. 4. Kelly detector: $P G$ for contaminated signal $N=2, P F=1 . D-10$.

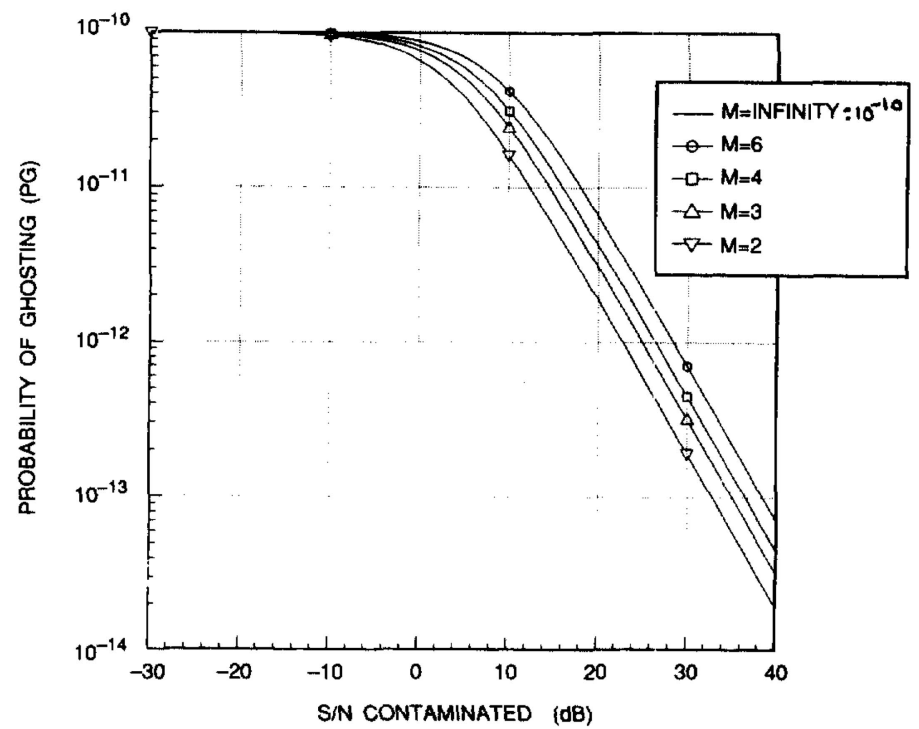

Fig. 5. Kelly detector: $P G$ for contaminated signal $N=30, P F=1 . \mathrm{D}-10$.

2) A small amount of contamination $\left((S / N)_{\text {con }}=\right.$ $-10 \mathrm{~dB}$ ) decreases $P_{G}$. However, $10 \mathrm{~dB}$ of contamination is necessary to make $P_{G}$ decrease approximately by a factor of 10 .

3) For most cases, $P_{D}$ begins to significantly decrease in 10 to $20 \mathrm{~dB}$ of contamination.

4) For small $M(2-6)$, the $P_{G} \mathrm{~s}$ are within a factor of five. This spread increases with increasing $N$.

5) As noted in [1], $P_{D}$ monotonically increases with increasing $M$. For $(S / N)_{\text {con }}=0$, the steady state $P_{D} \mathrm{~s}$ are indicated by the flat region of each curve.

6) For $P_{D}$, the GLRT and MLAD have similar relative performance trends.

One of the more significant results indicated by the curves is that the ghosting probability does not increase in the presence of contamination. Hence, the CFAR capability of both the GLRT and MLAD is not decreased in the sense that the false alarm probability (with contamination) is bounded from above by the quiescent false alarm probability (no contamination). However, because $P_{G}$ is decreasing, inherently the variable threshold of the detector (MLAD or GLRT) is increasing and, hence, the detection probability decreases.

One final note. A simple solution to decrease the effects of signal contamination is to use a large number of samples (make $K$ or $M$ large). However, we caution against this solution in that this was a simplified analysis where only one sample vector was contaminated. Obviously, if we take enough samples, the effects of this one sample can be significantly 


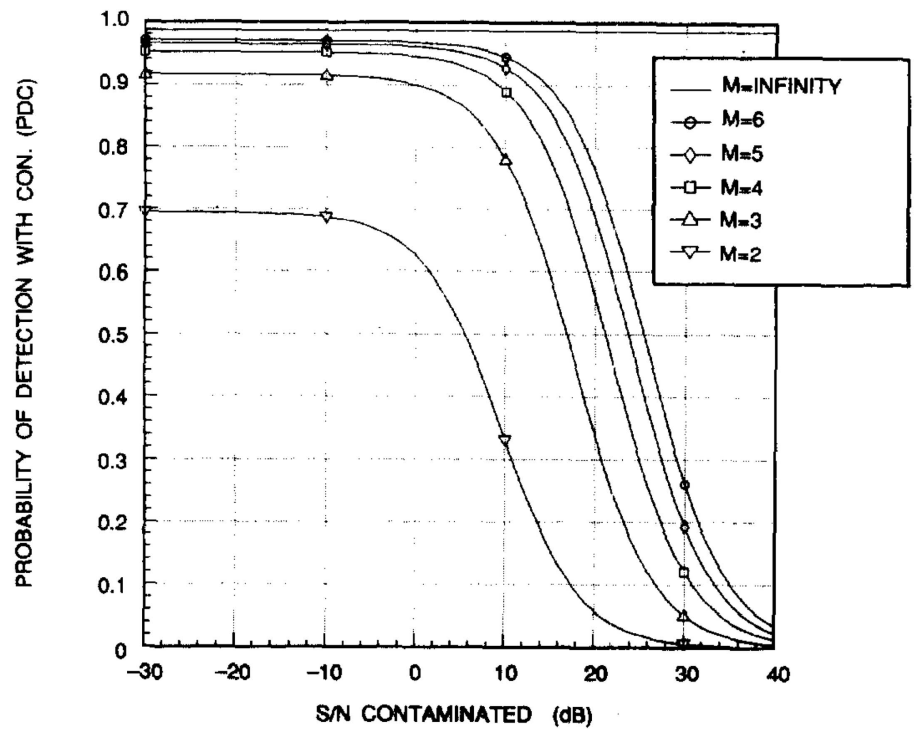

Fig. 6. Kelly detector: $P D$ for contaminated signal $N=2, P F=1 . D-6, S / N$ opt $=30 \mathrm{~dB}$.

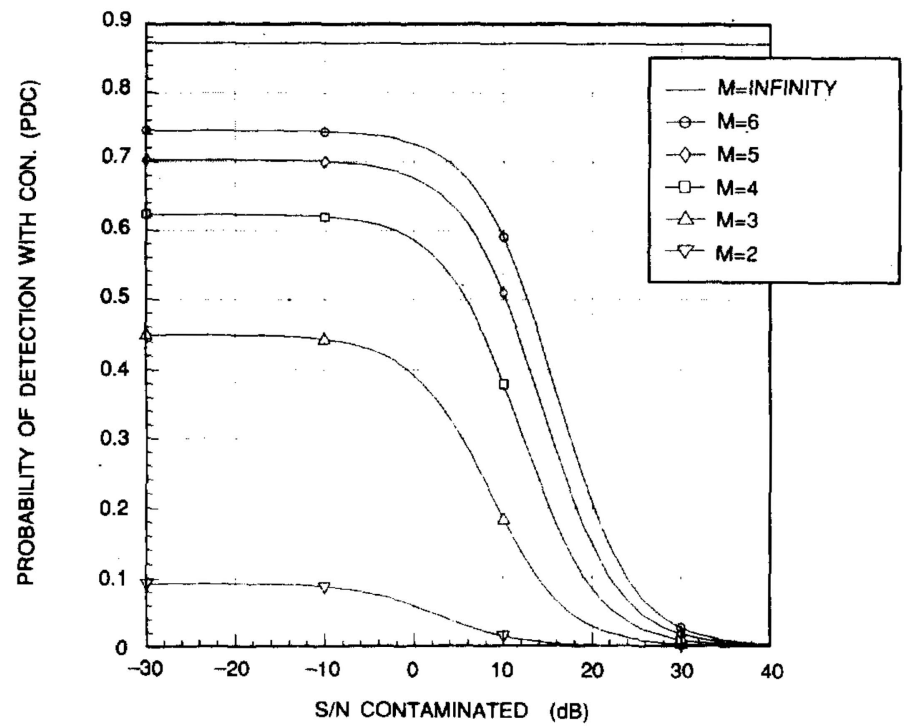

Fig. 7. Kelly detector: $P D$ for contaminated signal $N=2, P F=1 . D-6, S / N$ opt $=20 \mathrm{~dB}$.

diminished by averaging over many samples. In reality, making $K$ large can result in even more signal contamination, since there may be more opportunities for this to occur.

\section{SUMMARY}

Two schemes for adaptive detection, Kelly's GLRT and the MLAD, have been analyzed with respect to the deleterious effect of desired-signal contamination of the data used to compute the sampled covariance matrix for the two detections. This effect can occur when more than one desired signal is present in the sampled data. Detection probability $P_{D}$ and false alarm performance (ghosting probability $P_{G}$ ) were predicted for the two schemes under the assumptions that the input noises were Gaussian RVs that were temporally independent but spatially correlated; and the amplitude of the desired signal was Rayleigh distributed. $P_{D}$ and $P_{G}$ were computed as a function of the false alarm probability with no contamination $P_{F}$, the number of input channels, the number of independent samples-per-channel, the matched filtered output $S / N$ power ratio, and the $S / N$ of the contaminating desired signal. The $P_{D}$ and $P_{F}$ were obtained for a number of representative cases.

It was found that both $P_{D}$ and $P_{G}$ decreased with increasing levels of contamination. The $P_{G}$ performance was almost identical for the GLRT and MLAD. The $P_{D}$ performance for the two adaptive detectors showed similar relative performance trends. Significantly, it was shown that the ghosting probability 


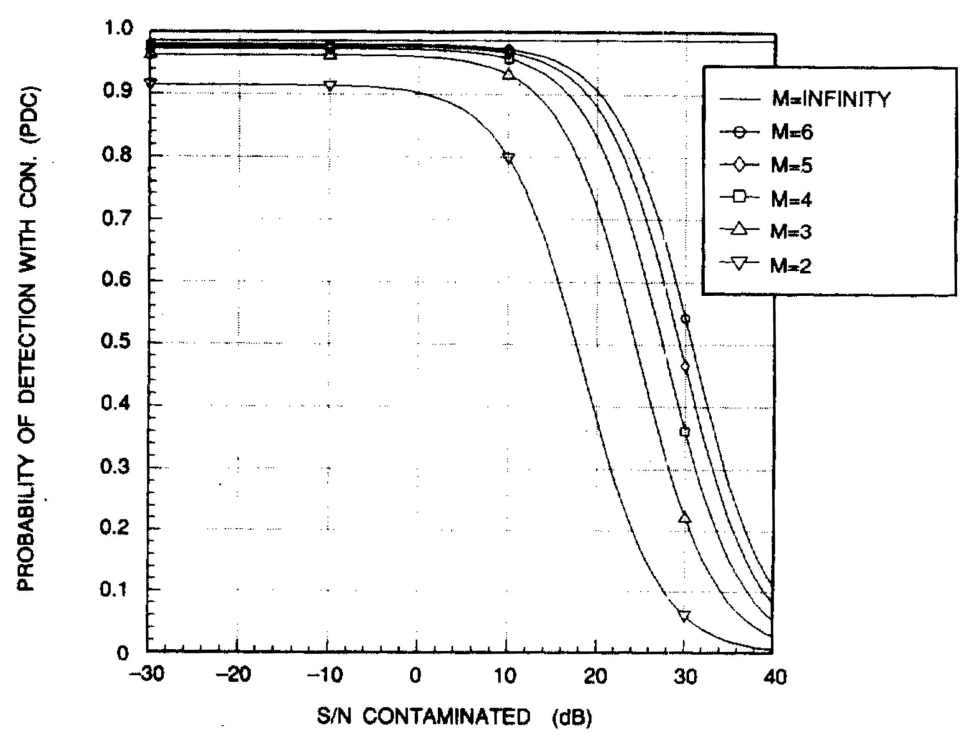

Fig. 8. Kelly detector: $P D$ for contaminated signal $N=5, P F=1 . D-6, S / N$ opt $=30 \mathrm{~dB}$.

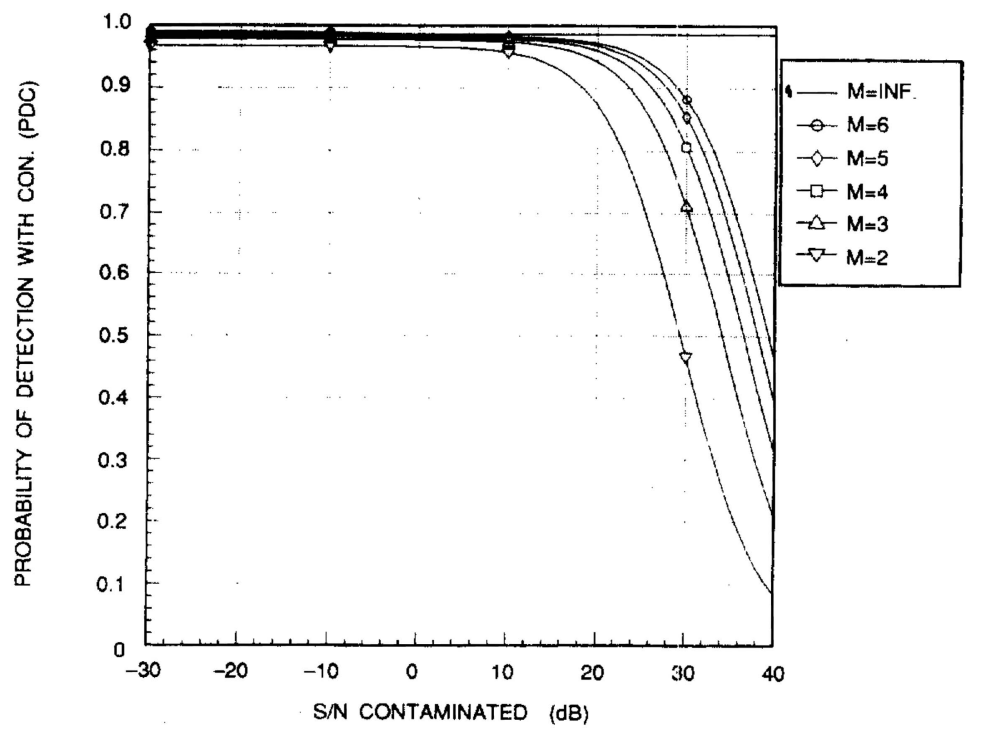

Fig. 9. Kelly detector: $P D$ for contaminated signal $N=30, P F=1 . D-6, S / N$ opt $=30 \mathrm{~dB}$.

does not exceed $P_{F}$ in the presence of contamination. Hence, the CFAR capability of the GLRT or MLAD is not degraded in the sense that false alarm probability is bounded from above by the quiescent false alarm probability (no contamination).

\section{APPENDIX. THE PROBABILITY DENSITY FUNCTION} OF $\eta$

Starting with (11), we write

$$
\eta=u_{22}^{2}=\sum_{k=N}^{K-1}\left|z_{N K}^{(N-1)}\right|^{2}+\left|z_{N K}^{(N-1)}\right|^{2}
$$

where $z_{N k}^{(N-1)}, k=N, \ldots, k-1$ are IID circular Gaussian RV with power equal to 1 , and $z_{N K}^{(N-1)}$ is a circular Gaussian RV with power equal to $\sigma_{c}^{2}+1$. Define

$$
\begin{aligned}
& x=\left|z_{N K}^{(N-1)}\right|^{2} \\
& y=\sum_{k=N}^{K-1}\left|z_{N k}^{(N-1)}\right|^{2}
\end{aligned}
$$

and

$$
L=K-N \text {. }
$$

The pdfs of $x$ and $y$ are given by

$$
\begin{aligned}
& p_{x}(x)=\frac{1}{\sigma_{c}^{2}+1} \exp \left\{-\frac{x}{\sigma_{c}^{2}+1}\right\} ; \quad x \geq 0 \\
& p_{y}(y)=\frac{1}{(L-1) !} y^{L-1} e^{-y} ; \quad y \geq 0 .
\end{aligned}
$$




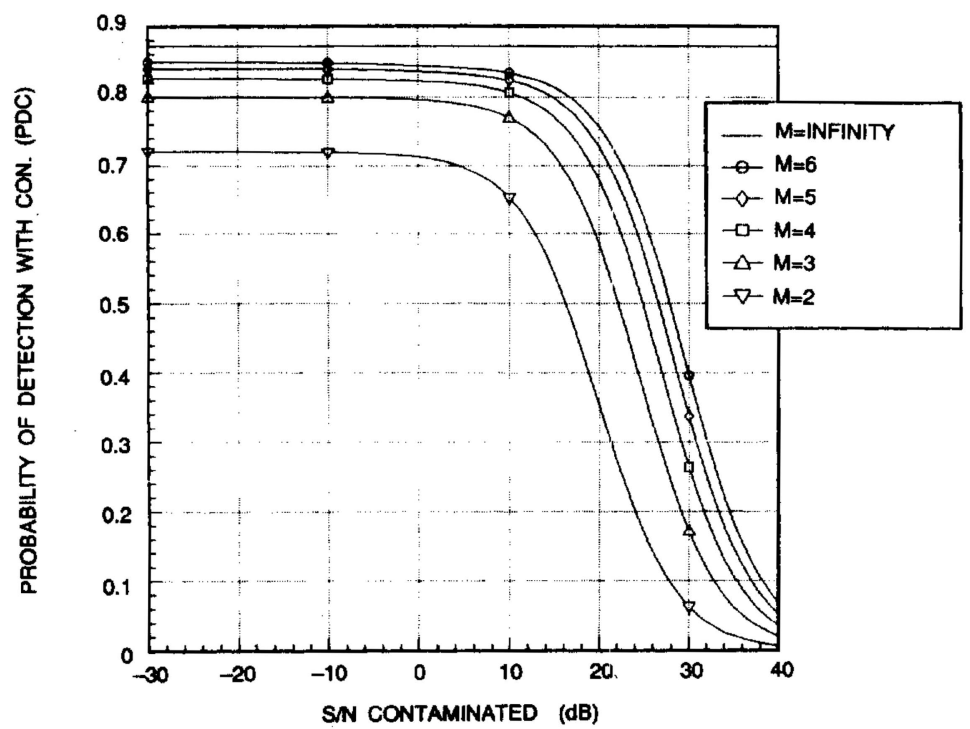

Fig. 10. Kelly detector: $P D$ for contaminated signal $N=30, P F=1 . D-6, S / N$ opt $=20 \mathrm{~dB}$.

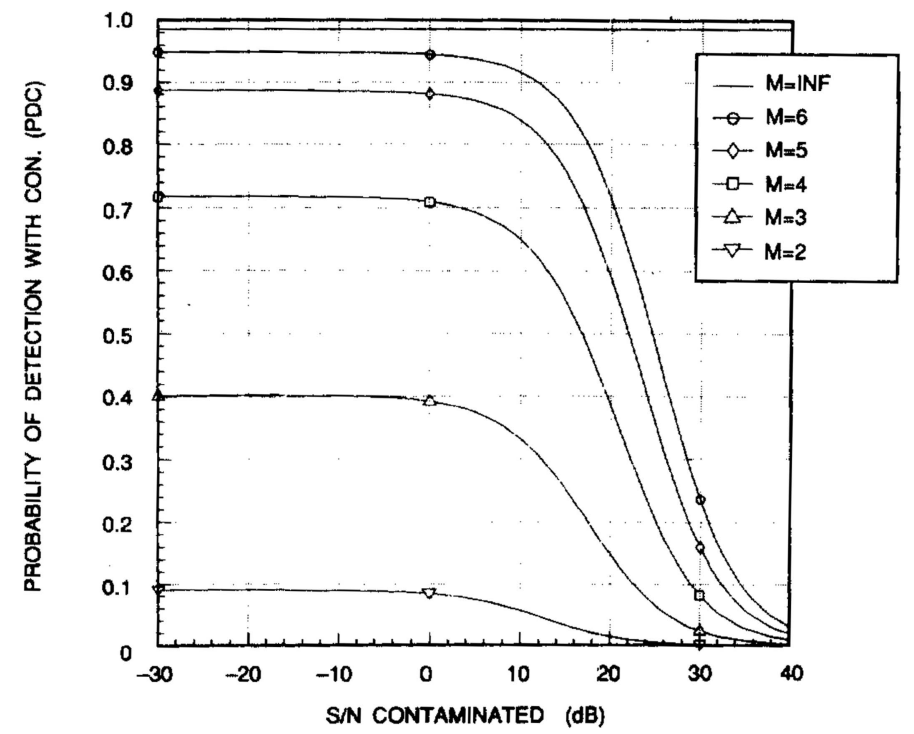

Fig. 11. MLD: $P D$ for contaminated signal $N=2, P F=1 . D-6, S / N$ opt $=30 \mathrm{~dB}$.

Now

$$
\begin{aligned}
p_{\eta}(\eta)= & \int_{0}^{\eta} p_{x}(\eta-\alpha) p_{y}(\alpha) d \alpha \\
= & \frac{1}{(L-1) !\left(\sigma_{c}^{2}+1\right)} \exp \left\{-\frac{\eta}{\sigma_{c}^{2}+1}\right\} \int_{0}^{\eta} \alpha^{L-1} \\
& \times \exp \left\{-\frac{\alpha \sigma_{c}^{2}}{\sigma_{c}^{2}+1}\right\} d \alpha .
\end{aligned}
$$

Set $\lambda=\alpha / \eta$. Then

$$
\begin{aligned}
p_{\eta}(\eta)= & \frac{1}{(L-1) !\left(\sigma_{c}^{2}+1\right)} \eta^{L} \exp \left\{-\frac{\eta}{\sigma_{c}^{2}+1}\right\} \int_{0}^{1} \lambda^{L-1} \\
& \times \exp \left\{-\frac{\lambda \eta \sigma_{c}^{2}}{\sigma_{c}^{2}+1}\right\} d \lambda .
\end{aligned}
$$

\section{REFERENCES}

[1] Gerlach, K. (1994)

A comparison of two adaptive detection schemes. IEEE Transactions on Aerospace and Electronic Systems, 30, 1 (Jan. 1994), 30-40.

[2] Kelly, E. J. (1986) An adaptive detection algorithm. IEEE Transactions on Aerospace and Electronic Systems, AES-22, 1 (1986), 115-127.

[3] Steenson, B. O. (1968)

Detection performance of a mean-level threshold. IEEE Transactions on Aerospace and Electronic Systems, AES-4, 4 (1968), 529-534.

[4] Dillard, G. M. (1974)

Mean-level detection of nonfluctuating signals.

IEEE Transactions on Aerospace and Electronic Systems, AES-10, 6 (1974), 795-799. 


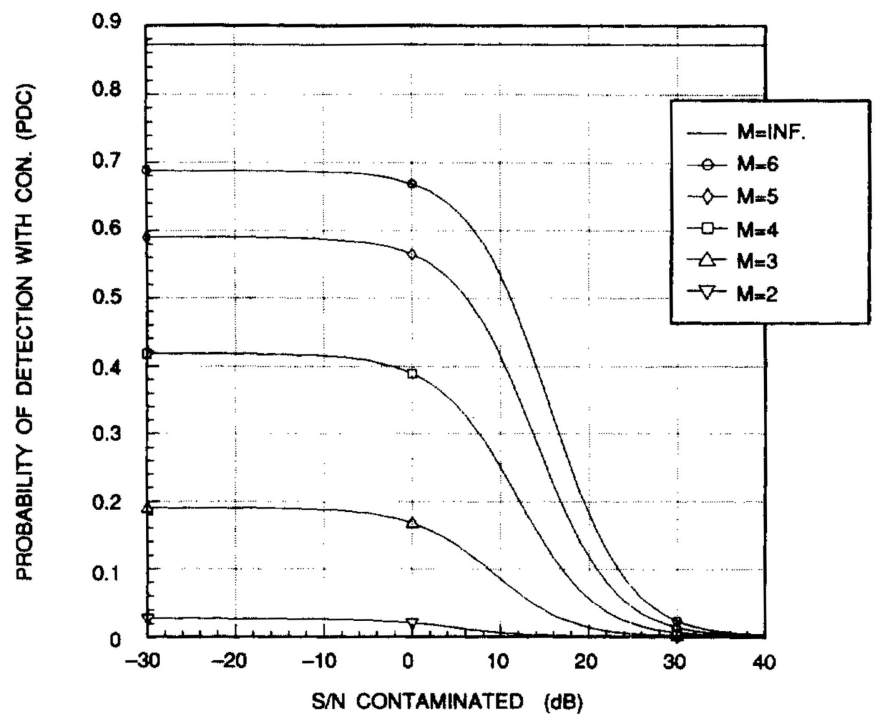

Fig. 12. MLD: $P D$ for contaminated signal $N=2, P F=1 . D-6, S / N$ opt $=20 \mathrm{~dB}$.

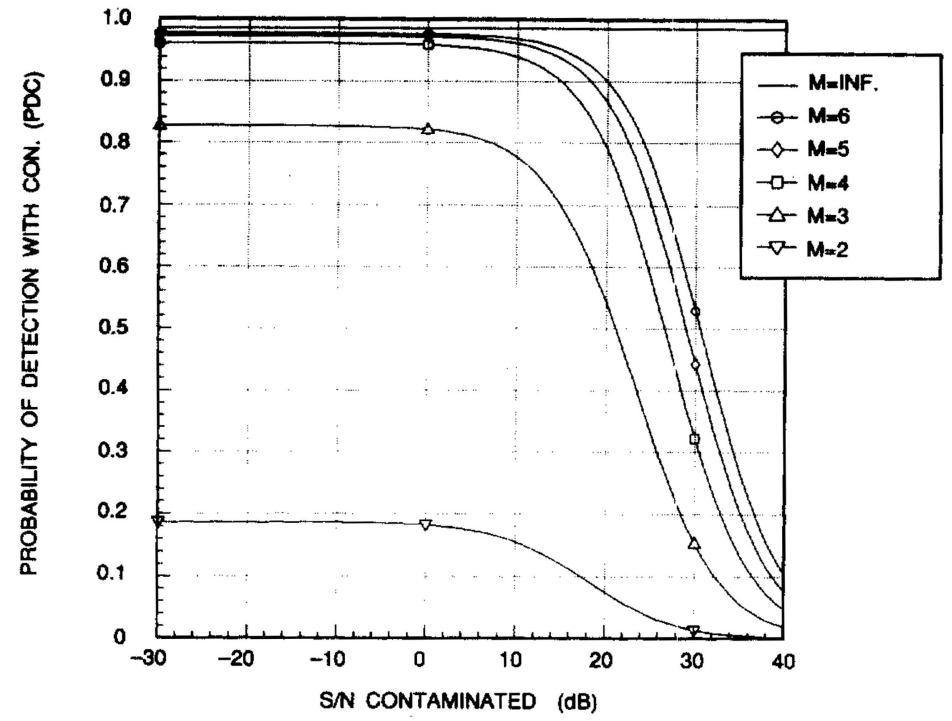

Fig. 13. MLD: $P D$ for contaminated signal $N=5, P F=1 . D-6, S / N$ opt $=30 \mathrm{~dB}$.

[5] Gerlach, K. (1994)

A mean level adaptive detector using nonconcurrent data. IEEE Transactions on Aerospace and Electronic Systems, 30, 1 (Jan. 1994), 258-266.

[6] Cai, L., and Wang, H. (1991)

Performance comparisons of the modified SMI and GLR algorithms.

IEEE Transactions on Aerospace and Electronic Systems, 27, 3 (May 1991), 487-491.
[7] Reed, I. S., Mallett, J. D., and Brennan, L. E. (1974) Rapid convergence rate in adaptive arrays. IEEE Transactions on Aerospace and Electronic Systems, AES-10, 6 (1974), 853-863.

[8] Di Franco, J. V., and Rubin, W. L. (1980) Radar Detection.

Dedham, MA: Artech House, 1980. 


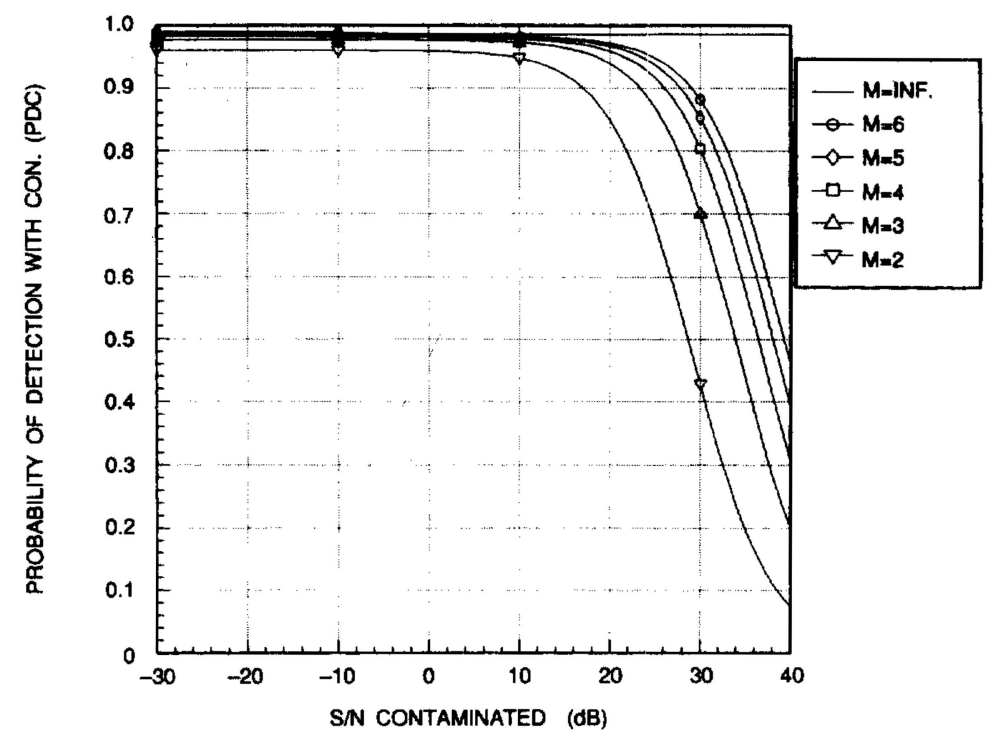

Fig. 14. MLD: $P D$ for contaminated signal $N=30, P F=1 . D-6, S / N$ opt $=30 \mathrm{~dB}$.

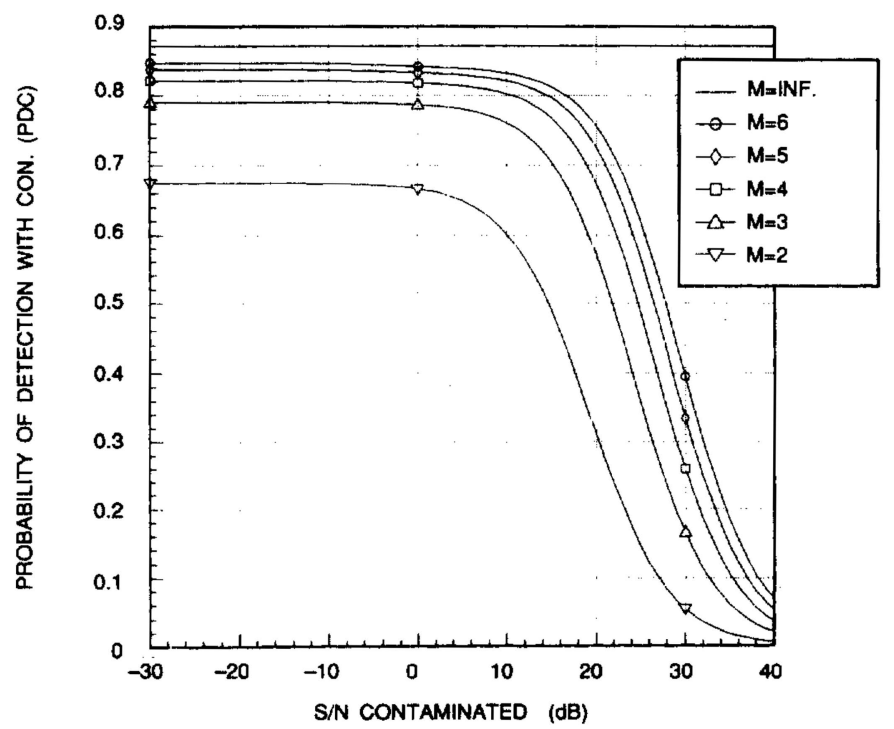

Fig. 15. MLD: $P D$ for contaminated signal $N=30, P F=1 . D-6, S / N$ opt $=20 \mathrm{~dB}$.

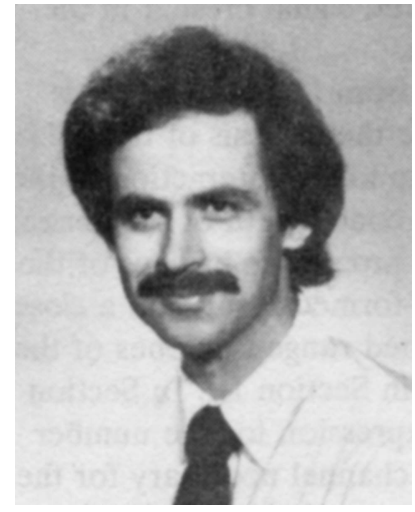

Karl Gerlach (M'81) was born in Chicago, IL. He received his B.S. in 1972 from the University of Illinois, Urbana, and his M.S. and D.Sc. from George Washington University, Washington, DC, in 1975 and 1981, respectively. All degrees are in electrical engineering.

Since 1972, he has been employed by the Naval Research Laboratory in Washington, DC. From 1972 to 1976, he worked on experimental submarine communications systems and from 1976 to the present he has been with the Radar Division where his research interests include adaptive signal processing and space-based radar.

Dr. Gerlach was the 1986 recipient of the IEEE AESS Radar Systems Panel award. 